TRANSACTIONS OF THE

AMERICAN MATHEMATICAL SOCIETY

Volume 235, January 1978

\title{
HOMOTOPY AND UNIFORM HOMOTOPY
}

BY

ALLAN CALDER AND JERROLD SIEGEL

\begin{abstract}
It is shown that the sets, homotopy and uniform homotopy classes of maps from a finite dimensional normal space to a space of finite type with finite fundamental group, coincide. Applications of this result to the study of remainders of Stone-Cech compactifications, Kan extensions, and other areas are given.
\end{abstract}

In this paper we consider the question of when the existence of homotopies implies existence of uniform homotopies for given maps of one space to another. Not surprisingly, the positive results we obtain require strong hypotheses on the spaces and maps in question. The domain must be finite dimensional normal and the codomain must have the homotopy type of a $\mathrm{CW}$-complex of finite type with finite fundamental group. However, as will be seen below, these hypotheses are sufficiently broad so as to admit interesting applications.

A major stumbling block to carrying out an investigation of when homotopic maps must also be uniformly homotopic seems to have been the example of the real line and the circle. While all maps of the real line to the circle are homotopic, a simple computation shows that there are uncountably many uniform homotopy classes of such maps [7]. What we show below is that this example is the exceptional case, the culprit being the circle. We prove the following:

THEOREM 1. Let $X$ be a finite dimensional normal space. Let $Y$ be a compact space with the homotopy type of a $C W$-complex and with $\pi_{1}(Y)$ finite. Then two maps $f, g: X \rightarrow Y$ are homotopic iff they are uniformly homotopic.

Indeed, in the paper itself we prove a somewhat stronger theorem, relaxing the hypothesis on $Y$ in various ways.

This result established, we proceed to a series of applications, somewhat unrelated in statement, but all depending heavily on a form of Theorem 1. We give two examples.

Received by the editors June 24, 1976.

AMS (MOS) subject classifications (1970). Primary 55E05; Secondary 54D30, 55B05.

Key words and phrases. Homotopy, uniform homotopy, Stone-Cech remainder, Kan extension, Cech extension, obstruction theory, bounded path length.

- American Mathematical Society 1978 
THEOREM 2. Let $M^{n}$ be a compact Riemannian manifold with $\pi_{1}\left(M^{n}\right)$ finite. Let $f, g: X \rightarrow M^{n}$ be homotopic maps of a finite dimensional normal space into $M^{n}$. Then $f$ and $g$ are homotopic via a uniform homotopy $H$ such that:

(1) For each $x \in X, H_{x}: I \rightarrow M^{n}$ is a smooth path.

(2) \{length $\left.\left(H_{x}\right) \mid x \in X\right\}$ is bounded.

(3) speed $\left.\left(H_{x}\right) \mid x \in X\right\}$ is bounded.

Note that uniform homotopy and bounded path length homotopy are logically independent.

THEOREM 3. Čech cohomology (finite covers) $H_{f}^{n}$ is a homotopy type invariant for the category of finite dimensional normal spaces iff $n \neq 1$. Moreover, $H_{f}^{2}$ is a homotopy invariant for all normal spaces.

The plan of the paper is as follows: In the first section, we give a new form of the Eilenberg-Steenrod definition of uniform homotopy [8] and establish some of its elementary properties. In particular we show that if the codomain is a compact metric space, then the definition is equivalent to Dowker's [7].

$\$ \S 2$ and 3 are devoted to a proof of a technical result we call the Bounded Lifting Lemma. This result is central to most of what follows and seems to be of independent interest.

In $\S 4$ we study the map $\beta: X \rightarrow \beta X$, the embedding of a space $X$ into its Stone-Čech compactification. More explicitly, we look at the map $\beta^{*}:[\beta X$, $Y] \rightarrow[X, Y]$ induced by $\beta$ on homotopy classes.

Since $[\beta X, Y]$ is essentially uniform homotopy classes of bounded (compact image) maps $X$ to $Y$ we have that the set of homotopy classes is naturally equivalent to the set of uniform homotopy classes iff $\beta^{*}$ is an isomorphism.

This is shown to be the case when $X$ is a finite dimensional normal space and $Y$ has the homotopy type of a $C W$-complex of finite type with $\pi_{1}(Y)$ finite. If $\pi_{1}(Y)$ is not finite, but we assume $Y$ is a loop space, then we show that there is a split short exact sequence of groups

$$
0 \rightarrow D(X, Y) \rightarrow[\beta X, Y] \stackrel{\beta^{*}}{\rightarrow}[X, Y] \rightarrow 0
$$

where $D(X, Y)$ is a direct sum of copies of $Q$ (the rationals).

In $\$ 5$ we consider applications of the above results to the study of Stone-Čech remainders, $N X=\beta X-X$. NX carries information about the function algebra of $X$ [9] and the techniques used in this paper grew out of studies directed primarily at developing an understanding of $N X$ for certain spaces [1], [11].

In particular, we obtain the following curious theorem.

THEOREM 4. Let $M^{n}$ be a manifold with boundary $\partial M^{n}$. Let $N M^{n}=$ 
$\beta\left(\right.$ int $\left.M^{n}\right)-$ int $M^{n}$ be its Čech boundary. Then $[\partial M, Y] \simeq\left[N M^{n}, Y\right]$ for all $Y$ as in Theorem 1 above.

In $\S 6$ we study Čech and Kan extensions of functors en route proving Theorem 3 above.

In $\$ 7$ we relate homotopy and bounded path length homotopy. Here the main result is Theorem 2 above.

Finally, in $\$ 8$ we discuss the obstruction theoretic question of when a map $f: \beta X \times A \rightarrow Z$ extends to a map $\beta X \times Y \rightarrow Z$, where $(Y, A)$ is a relative $C W$-complex $Z$ is a loop-space and it is known that $f \mid X \times A$ extends to $X \times Y$. Note, when $(Y, A)=(I,\{0,1\})$ this is the question as to when a homotopy is uniform. Obstruction cohomology classes $\theta^{i} \in H^{i}\left(Y, A ; G_{i}\right)$ are presented where $G_{i}$ is a suitable direct sum of copies of $Q$. As expected, $\theta^{i}$ vanishes if and only if it is possible to extend the map from $\beta X \times Y^{i-1}$ to $\beta X \times Y^{i}$, where $Y^{i}$ is the $i$ th skeleton of $(Y, A)$.

1. Preliminaries. In this section we gather together various results that we will use in the following sections.

(1.1) All spaces will be assumed to be Hausdorff.

The category of normal spaces and maps (= continuous functions) and its full subcategory of compact spaces will be denoted by $N$ and $C_{2}$, respectively.

The Stone- $\check{C}$ ech functor $\beta: N \rightarrow C_{2}$ sends $X \in N$ to its Stone-Čech compactification $\beta X$ and $f: X \rightarrow Y$ to the unique extension $\beta(f): \beta X \rightarrow \beta Y$. We shall also use $\beta: X \rightarrow \beta X$ to denote the dense embedding of $X$ into $\beta X$.

A map will be called bounded if its image is contained in a compact subset of its codomain. Hence, $f: X \rightarrow Y$ is bounded iff it factors through the diagram.

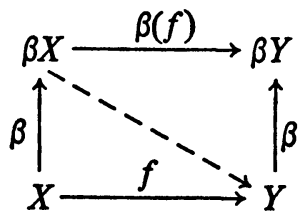

The dotted arrow, if it exists, is, of course, unique and will, without confusion, be denoted by $\beta(f)$ also.

The notion of uniform homotopy has several equivalent formulations. Our techniques have suggested the introduction of the following definition which is equivalent to the usual ones as we shall see in (1.3).

(1.2) Definimion. Let $X$ be an arbitrary space, $Y \in N$ and $I$ denote the unit interval. A map $H: X \times I \rightarrow Y$ is called a uniform homotopy iff the associated map

$$
h: X \rightarrow(\beta Y)^{I}, \quad h(x)(t)=\beta \circ H(x, t),
$$


is bounded, where $(\beta Y)^{I}$ is the space of functions from $I$ to $\beta Y$ with the compact-open topology.

Two maps $f, g: X \rightarrow Y$ will be called uniformly homotopic $\left(f \sim_{\beta} g\right)$ iff there is a uniform homotopy $H: X \times I \rightarrow Y$ with $H(x, 0)=f(x)$ and $H(x$, 1) $=g(x)$ for all $x \in X$.

(1.3) Lemma. The following are equivalent for $X \in N$ :

(a) $H: X \times I \rightarrow Y$ is a uniform homotopy.

(b) $H$ extends to a map $\bar{H}: \beta X \times I \rightarrow \beta Y$ (note $\beta X \times I \neq \beta(X \times I)$ ).

(c) For every finite open cover $U$ of $Y$ there exist a finite open cover $V$ and $W$ of $X$ and I, respectively, such that $V \times W$ refines $H^{-1} U$.

Further, if $Y$ is a compact metric space (a), (b), (c) are equivalent to

(d) for all $\varepsilon>0$ there exist $\delta>0$ such that $\left|t-t^{\prime}\right|<\delta$ implies $\mid H(x$, $t)-H\left(x, t^{\prime}\right) \mid<\varepsilon$ for all $x \in X$.

Proof. (a) $\Leftrightarrow(\mathrm{b}): h: X \rightarrow(\beta Y)^{I}$ is bounded iff it extends to a map $\bar{h}$ : $\beta X \rightarrow(\beta Y)^{I}$. Define $\bar{H}: \beta X \times I \rightarrow \beta Y$ by $\bar{H}(x, t)=\bar{h}(x)(t)$.

(b) $\Leftrightarrow$ (c): [8, p. 282, Theorem 9.9].

For $Y$ compact metric:

(c) $\Rightarrow$ (d): immediate.

(d) $\Rightarrow$ (b): since $Y$ is compact the map $H_{t}: X \rightarrow Y\left(H_{t}(x)=H(x, t)\right)$ can be extended to $\beta\left(H_{t}\right): \beta X \rightarrow Y=\beta Y$ for each $t \in I$. Define $\bar{H}: \beta X \times I \rightarrow Y$ by $\bar{H}(x, t)=\beta\left(H_{t}\right)(x)$. This function is always defined. The problem is to show that it is continuous. This follows from (d) by a standard " $\varepsilon / 3$ " proof.

Our technical results are actually of the form "homotopy implies a relation stronger than uniform homotopy". We now introduce that relation.

(1.4) Definition. If a map $h: X \rightarrow Y^{I}$ extends to $h: \beta X \rightarrow Y^{I}$ then we call the associated map $H: X \times I \rightarrow Y$ a strong uniform homotopy. We write $f \sim_{s \beta}$ $g$ for maps so related.

Clearly $H$ is a strong uniform homotopy iff $h(X)$ lies in a compact subset of $Y^{I}$. The following formulation of this observation will be used below.

Let $p: Y^{I} \rightarrow Y \times Y$ be the (Hurewicz) fibration given by $p(\sigma)=(\sigma(0)$, $\sigma(1))$. This fibration serves to characterize homotopy in the sense that two maps $f, g: X \rightarrow Y$ are homotopic iff there is a map $h: X \rightarrow Y^{I}$ such that

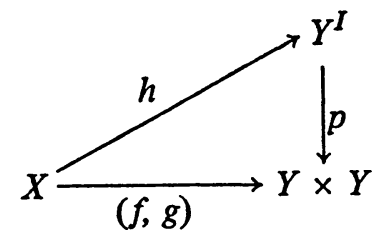

commutes.

Strong uniform homotopy for bounded maps may be characterized using this fibration as follows. 
(1.5) Lemma. Let $f, g: X \rightarrow Y$ be bounded maps ( $X$ and $Y \in N$ ). The following are equivalent:

(a) $f \sim_{s \beta} g$.

(b) There exists a bounded map $h: X \rightarrow Y^{I}$ such that

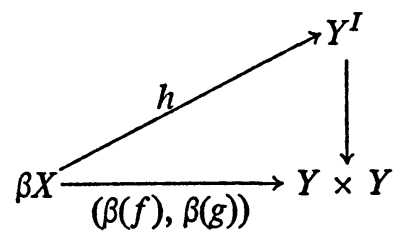

commutes.

We see from (1.5) that the set of strong uniform homotopy classes of bounded maps from $X$ to $Y$ is the "same" as the set $[\beta X, Y]$ of homotopy classes of maps of $\beta X$ to $Y$. Thus the map

$$
\beta^{*}:[\beta X, Y] \rightarrow[X, Y],
$$

induced by $\beta: X \rightarrow \beta X$, may be used to measure the difference between homotopy classes and strong uniform homotopy classes of bounded maps.

For reasonable hypothesis (e.g. $Y$ compact) $\beta^{*}$ is easily seen to be onto. That it is not in general 1-1 is shown by the following result of Dowker [7].

(1.7) Lemma. For $X \in N$ and $S^{1}$ the unit circle,

$$
\operatorname{ker}\left\{\beta^{*}:\left[\beta X, S^{1}\right] \rightarrow\left[X, S^{1}\right]\right\} \cong C(X) / C^{*}(X),
$$

where $C(X)$ is the additive group of real valued functions of $X$ and $C^{*}(X)$ is the subgroup of bounded real valued functions.

Proof. Consider the following commutative diagram of groups

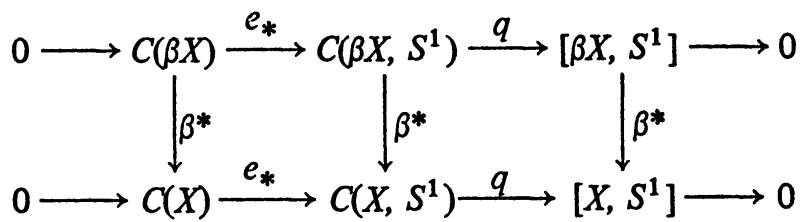

where $C\left(, S^{1}\right)$ is the group of continuous functions into $S^{1}$. The vertical maps are induced by $\beta$, the $e_{*}$ 's are induced by the exponential map $e$ : $R \rightarrow S^{1}$ and the $q$ 's are quotient maps.

Now two maps into $S^{1}$ are homotopic if and only if their difference factors through $e$. So the horizontal sequences are exact, and since $\beta^{*}: C(\beta X$, $\left.S^{1}\right) \rightarrow C\left(X, S^{1}\right)$ is an isomorphism (the universal property of $\beta$ ), we have

$$
\begin{aligned}
\operatorname{ker}\left\{\beta^{*}:\left[\beta X, S^{1}\right] \rightarrow\left[X, S^{1}\right]\right\} & =\operatorname{coker}\left\{\beta^{*}: C(\beta X) \rightarrow C(X)\right\} \\
& =C(X) / C^{*}(X) .
\end{aligned}
$$


Note $C(X) / C^{*}(X)$ is a divisible group without torsion.

In the following sections (1.7) will take an added significance as we will show that within a significant class of spaces this is essentially the only example where $\beta^{*}$ is not injective.

In several places in this paper we will make use of a somewhat special extension property. We now state it for reference.

(1.8) Lemma. Let $p: E \rightarrow B$ be a fibration (Hurewicz fibration). Let $X^{\prime} \subset X$ be a pair of topological spaces. Suppose we are given

(1) $f: X \rightarrow B$ and $g: X^{\prime} \rightarrow E$ with $p g=f / X^{\prime}$,

(2) $F$, a homotopy $f \sim f^{\prime}$ and $G$ a homotopy $g \sim g^{\prime}$ with $p G=F / X^{\prime} \times I$,

(3) $G^{\prime}$, a homotopy $g^{\prime} \sim \bar{g}^{\prime}$ with $p G^{\prime}=f^{\prime} / X^{\prime}$,

(4) $\tilde{g}^{\prime}: X \rightarrow E$ with $p \tilde{g}^{\prime}=f^{\prime}$ and $\tilde{g}^{\prime} / X^{\prime}=\bar{g}^{\prime}$.

Then there exists a map $\bar{g}: X^{\prime} \rightarrow E$ homotopic to $g$ over $f$ (i.e. fiberwise) and such that $\bar{g}$ admits an extension $\tilde{g}: X \rightarrow E$ with $p \tilde{g}=f$.

Proof. Let $F^{-1}: X \times I \rightarrow B$ be defined by $F^{-1}(x, t)=F(x, 1-t)$. Let $\tilde{G}: X \times I \rightarrow E$ cover $F^{-1}$ with initial position $\tilde{g}^{\prime}$. Let $\tilde{g}=\tilde{G}_{1}$ and $\bar{g}=\tilde{g} / A$. We now check that $\bar{g} \sim g$ over $f$. This involves a use of the absolute covering homotopy extension property to which we are restricting in this general setting.

Let $J=\{1\} \times I \cup I \times\{0,1\} \subset I \times I$. Let $\theta:(I \times I, J) \simeq(J \times I, J \times$ 0 ) be a homeomorphism of pairs. Let $\bar{F}: X^{\prime} \times I \times I \rightarrow B$ be defined by the formula $\bar{F}(a, s, t)=F(a, s)$.

Finally, define $\bar{G}=X^{\prime} \times J \rightarrow E$ by

$$
\bar{G}= \begin{cases}G & \text { on } X^{\prime} \times I \times\{0\}, \\ G^{\prime} & \text { on } X \times\{1\} \times I, \\ G^{\prime} \mid X^{\prime} & \text { on } X \times I \times\{1\} .\end{cases}
$$

Now cover $\bar{F} \theta^{-1}$ with initial map $\bar{G}$. Call the covering $H$. Then $H \theta$ covers $\bar{F}$ and $(H \theta)_{1}$ is the desired homotopy over $f$.

We also need the following lemma for reference.

(1.9) Lemma. Let $B$ be a finite simplicial complex. Let $f, f^{\prime}: X \rightarrow B$ be contiguous maps. Then the linear homotopy

$$
F(x, t)=t f^{\prime}(x)+(1-t) f(x)
$$

between $f$ and $f^{\prime}$ is uniform.

Proof. Recall that the topology on $B$ is given by the metric

$$
d(a, b)=\left(\sum_{\alpha}\left(\Pi_{\alpha}(a)-\Pi_{\alpha}(b)\right)^{2}\right)^{1 / 2}
$$


where $\left\{\Pi_{\alpha}\right\}$ are the barycentric coordinate functions. The proof is immediate from (1.3)(d).

2. The bounded lifting property. In the first section we reduced the question of existence of strong uniform homotopies to the question of when a map can be lifted to a bounded map into the total space of a particular fibration. In this section we consider this second question in more generality. We will need the added generality in later sections.

(2.1) Notation. Let Top ${ }_{2}$ be the category of all Hausdorff spaces and maps and let $W, W_{f}, W_{f t}, W_{f d}$ denote the full subcategories of $\mathrm{Top}_{2}$ whose objects are respectively, all $C W$-complexes, all finite complexes, all $C W$-complexes of finite type (i.e. finitely many cells in each dimension) and all finite dimensional $C W^{\prime}$-complexes.

$\tilde{W}\left(\tilde{W}_{f t}\right)$ will denote the full subcategory of Top ${ }_{2}$ whose objects have the homotopy type of spaces in $W\left(W_{f t}\right)$. Finally, "fibration" $p: E \rightarrow B$ means Hurewicz fibration with base admitting a numerable cover $\left\{U_{\alpha}\right\}$ such that $p^{-1}\left(U_{\alpha}\right)$ is trivial in the sense of Dold [6].

(2.2) Definition. A fibration $p: E \rightarrow B$ is said to have the bounded lifting property with respect to a subcategory $A$ of $\operatorname{Top}_{2}(\operatorname{BLP}(A))$ if for each $X \in A$, bounded map $f: X \rightarrow B$, and map $g: X \rightarrow E$ such that

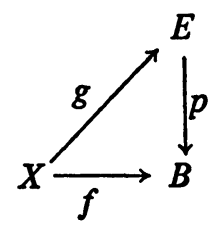

commutes, $g$ is homotopic over $f$ to a bounded map $\bar{g}: X \rightarrow E$. That is, there is a homotopy $H: X \times I \rightarrow E$ such that $H_{0}=g, H_{1}=\bar{g}$ and $p H(x, t)=f(x)$ for all $t \in I$.

We now prove a result which is a prototype for our Bounded Lifting Lemma of the next section and which will find an independent application in $\$ 6$.

(2.3) LeMma. Let $p: E \rightarrow B$ be a fibration. If $p$ has a fiber which is homotopic to a compact space $F$ then it has $\mathrm{BLP}\left(\mathrm{Top}_{2}\right)$.

Proof. Let $f$ and $g$ be as in (2.2). By [6] there exists an open cover $\left\{U_{i}\right\}_{i=1}^{n}$ of $\overline{f(X)}$ such that $p^{-1}\left(\bar{U}_{i}\right)$ is fiber homotopy equivalent to $\bar{U}_{i} \times F$. Let

$$
p^{-1}\left(U_{i}\right) \underset{\psi_{i}}{\stackrel{\phi_{i}}{\rightleftarrows}} \bar{U}_{i} \times F
$$

be a fiber homotopy equivalence and its inverse. Let $\left\{V_{i}\right\}_{i=1}^{n}$ be a cover of $\overline{f(X)}$ such that $\bar{V}_{i} \subset U_{i}$ and $\bar{V}_{i}$ is compact. Note $\overline{f(X)}$ is compact. 
Let $H_{i}: p^{-1}\left(\bar{U}_{i}\right) \times I \rightarrow p^{-1}\left(\bar{U}_{i}\right)$ be a fiber homotopy from $\psi_{i}$, $\phi_{i}$ to the identity and let $\underline{\eta}_{i}: B \rightarrow I$ be a map such that $\eta_{i} \mid \bar{V}_{i}=0$ and $\eta_{i} \mid\left(B-U_{i}\right)=1$.

Let $K_{1}=\psi_{1}\left(\bar{V}_{1} \times F\right)$. Then $K_{1}$ is compact and $\psi_{1} \phi_{1} g\left(f^{-1} \bar{V}_{1}\right) \subset K_{1}$.

Define $g_{1}: X \rightarrow E$ by

$$
g_{1}(x)= \begin{cases}\psi_{1} \phi_{1} g(x), & f(x) \in \bar{V}_{1}, \\ g(x), & f(x) \notin U_{1}, \\ H_{1}\left(g(x), \eta_{1} f(x)\right) & \text { otherwise. }\end{cases}
$$

Then $g_{1} \sim g$ over $f$ and $g_{1}\left(f^{-1}\left(\bar{V}_{1}\right)\right) \subset K_{1}$.

Now suppose we have $K_{i} \subseteq E$ and $g_{i}$ such that $K_{i}$ is compact, $g_{i}\left(\cup_{j<i} \bar{V}_{j}\right)$ $\subset K_{i}$ and $g_{i} \sim g$ over $f$.

Define

$$
K_{i+1}=\psi_{i+1}\left(\bar{V}_{i+1} \times F\right) \cup K_{i} \cup H_{i+1}\left(\left(K_{i} \cap p^{-1} \bar{U}_{i+1}\right) \times I\right)
$$

and $g_{i+1}: X \rightarrow E$ by

$$
g_{i+1}(x)= \begin{cases}\psi_{i+1} \phi_{i+1} g_{i}(x), & f(x) \in \bar{V}_{i+1}, \\ g_{i}(x), & f(x) \notin U_{i+1}, \\ H_{i+1}\left(g_{i}(x), \eta_{i+1} f(x)\right) & \text { otherwise. }\end{cases}
$$

The result follows by induction on $i$.

The next proposition is the key technical result of the paper. It essentially gives conditions under which one may deform a homotopy into a strong uniform homotopy. The proof itself is quite long and occupies the remainder of this section. The next section also is devoted to further extensions of the result.

(2.4) Proposition. Let $p: E \rightarrow B$ be a fibration with $B$ having the homotopy type of a $C W$-complex. Let the fiber $F$ be a complex of finite type. Then the fibration has the $\operatorname{BLP}\left(S_{f d}\right)$, where $S_{f d}$ is the category of simplicial complexes.

Proof. The proof begins with some general observations which reduce the proof of the proposition to the case where $B$ is a finite simplicial complex and $f$ a simplicial map.

(2.5) LEMMA. Suppose the proposition holds for a space $B^{\prime}$ homotopy equivalent to $B$; then it holds for $B$.

Proof. Let

$$
B \underset{\psi}{\stackrel{\phi}{\rightleftarrows}} B^{\prime}
$$


be the homotopy equivalence. Consider the pullback

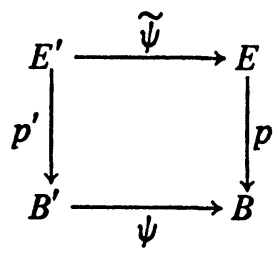

Then $p^{\prime}$ is a fibration with fiber $F$, hence, has the $\operatorname{BLP}\left(S_{f d}\right)$.

Let $\tilde{\phi}: E \rightarrow E^{\prime}$ be a fiber homotopy inverse to $\tilde{\psi}$ (see 2.1 and [5]). $\tilde{\phi}$ is defined by the diagram

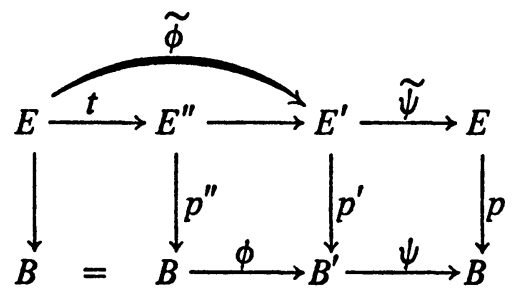

where $t$ is a fiber homotopy equivalence. Now let $f$ and $g$ be as in (2.2). Since $f$ is bounded so is $\phi f$, and $\tilde{\phi} g$ is a lift of $\phi f$. We now apply the bounded lifting property to deform $\tilde{\phi} g$ over $\phi f$ to a bounded map $g^{\prime}: X \rightarrow E^{\prime}$.

Now apply $\psi$ and $\tilde{\psi}$ to obtain a homotopy over $\psi \phi f$ of $\tilde{\psi} \tilde{\phi} g$ to a bounded map $\psi g^{\prime}$. Remembering that bounded "means" extendable to $\beta X$ we apply (1.8) to find a bounded map $\bar{g}$ homotopic to $g$ over $f$. In order to apply (1.8) one need only observe that the homotopy $\psi \phi f \sim f$ extends trivially. $\psi \phi \beta(f) \sim$ $\beta(f)$, being composition of $\beta(f)$ with the homotopy $\psi \phi \sim$ Identity.

By (2.5) we may assume $B$ is actually a $C W$-complex. In fact, by [12, Chapter 4, §4] for example, we may assume that $B$ is a simplicial complex.

Finally, since in the definition of the $\operatorname{BLP}\left(S_{f d}\right) f$ is bounded, it is clear that $E \rightarrow^{p} B$ has the property iff $p^{-1}(\bar{B}) \rightarrow^{p} \bar{B}$ has the property for every finite subcomplex $\bar{B}$.

We now show that we may assume $f$ is simplicial. Since after subdivision $f$ is contiguous to a simplicial map, this follows at once from (1.9) and the following lemma.

(2.6) Lemma. Let $p: E \rightarrow B$ be a fibration, $B$ a finite simplicial complex. Let $f, f^{\prime}: X \rightarrow B$ be uniformly homotopic where $X \in N$. Suppose the fibration has the $B L P$ over the map $f^{\prime}$ for $X$. Then it also has it over the map $f$.

Proof. (Again we use 1.8.) By (1.5) there exists a homotopy $F: \beta X \times I \rightarrow$ $B$ from $\beta(f)$ to $\beta\left(f^{\prime}\right)$. Let $G: X \times I \rightarrow E$ be a lift of $F \mid(X \times I)$ such that $G_{0}=g$. By assumption there is a bounded map $\bar{g}: X \rightarrow E$ homotopic over $f^{\prime}$ to $G_{1}$ covering the reverse of $F$; starting at $\beta \bar{g}$ we obtain a map $g: \beta X \rightarrow E$ 
over $f$. Now apply (1.8) to show $\tilde{g} \mid X$ is homotopic to $g$ over $f$.

(2.7) Continuation of THE PROOF of 2.4. Let $\left\{U_{k}\right\}$ be the closed star covering of $B$.

We have $p^{-1} U_{k}$ fiber homotopy equivalent to $U_{k} \times F$. We write

$$
p^{-1} U_{k} \underset{\psi_{k}}{\stackrel{\phi_{k}}{\rightleftarrows}} U_{k} \times F
$$

where $\phi_{k}$ is a fiber homotopy equivalence and $\psi_{k}$ is an inverse for $\phi_{k}$.

Let $H_{k}: p^{-1} U_{k} \times I \rightarrow p^{-1} U_{k}$ be a fiber homotopy from the identity to $\psi_{k} \phi_{k}$.

(2.8) The proof now proceeds by induction on the skeleta of $X$. The induction step is two stage. Assuming the result for a given skeleton, we first extend to a neighborhood of that skeleton and then to the next higher skeleton.

Note that, since $f$ is simplicial, for each closed simplex $\bar{\sigma}$ of $X, f(\bar{\sigma}) \subset U_{k}$ for some $k$.

Let $X^{i}$ denote the $i$ th skeleton of $X$ and $X_{A}^{i-1}$ denote the annular neighborhood of $X^{i-1}$ in $X^{i}$ given by $X_{A}^{i-1} \cap \bar{\sigma}^{i}=\left\{x \in \bar{\sigma}^{i}:\|x\|>\frac{1}{2}\right\}$ for each $i$-simplex, $\bar{\sigma}^{i}$, in $X^{i}$, the norm $\|x\|$ being given in the usual way by the barycentric coordinates of $\overline{\boldsymbol{\sigma}}^{i}$.

(2.9) Lemma. Suppose that there exists a compact set $K^{i-1}$ with $g\left(X^{i-1}\right) \subset$ $K^{i-1}$. Then we can find a compact set $K_{A}^{i-1}$, an annular neighborhood $X_{A}^{i-1}$ of $X^{i-1}$ in $X^{i}$, and a fiberwise deformation of $g$ to $g_{A}$ so that:

(1) $g_{A}\left(X_{A}^{i-1}\right) \subset K_{A}^{i-1}$,

(2) $g_{A}=g$ on $X^{i-1}$ (constant during deformation).

Proof. Denote the top dimensional cells of $B$ by $\Delta_{k}$, so for each $\alpha$ there exists a $k$ with $p g\left(\bar{\sigma}_{\alpha}\right) \subset \Delta_{k}$.

We first define $K_{A}^{i-1}$. For each $k$ let

$$
S_{k}: \Delta_{k} \times \Delta_{k} \times I \rightarrow \Delta_{k} \subset B
$$

be the usual linear homotopy such that

$$
S_{k}\left(x_{0}, x_{1}, 0\right)=x_{0}, \quad S_{k}\left(x_{0}, x_{1}, 1\right)=x_{1} .
$$

$S_{k}$ is a homotopy of $\pi_{0}$ to $\pi_{1}: \Delta_{k} \times \Delta_{k}$ to $\Delta_{k}$.

Let $K_{k}^{i-1}$ be the pull back of the diagram

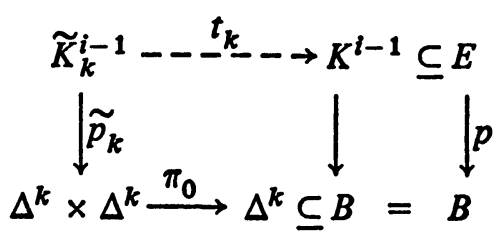


Now cover the homotopy $S_{k} \tilde{p}_{k}: \tilde{K}_{k}^{i-1} \times I$. Since the fibration is regular [20] ( $B$ a finite complex) we may assume the covering homotopy is constant over constant paths. Call this homotopy $\bar{S}_{k}$.

Define $K_{k}^{i-1}=\bar{S}_{k}\left(\tilde{K}_{k}^{i-1} \times 1\right)$ and set $K_{A}^{i-1}=\cup_{k}\left(K_{k}^{i-1}\right)$.

Now let $\bar{\sigma}^{i}$ be an $i$-simplex of $X$ and assume $p g\left(\bar{\sigma}^{i}\right) \subseteq \Delta_{k}$. Let $\bar{\sigma}_{A}^{i-1}=\left\{x \in \bar{\sigma}^{i} \mid\|x\| \geqslant \frac{1}{2}\right\}$ and let $r: \sigma_{A}^{i-1} \rightarrow \sigma_{A}^{i-1}$ be radial projection to the boundary and define $g_{0}=g r$.

Now define

$$
W: \sigma_{A}^{i-1} \times I \rightarrow \Delta^{k} \text { by } W=S_{k}\left(\left(p g_{0}, p g\right) \times I\right) .
$$

$W$ is covered by $\bar{W}: \sigma_{A} \times I \rightarrow E, \bar{W}=\bar{S}_{k}\left(g_{0} \times I\right)$, and $g_{0}$ is defined by the following diagram:

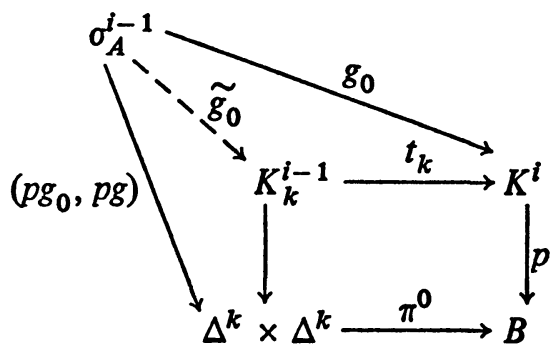

Finally, define $g_{A} \mid \bar{\sigma}_{A}^{i-1} \subset K_{A}^{i-1}$ by $g_{A}(x)=\bar{W}(x, 1)$. One checks

(a) $g_{A}$ covers $f$,

(b) $g_{A}\left|\dot{\sigma}^{i}=g\right| \dot{\sigma}^{i}\left(\dot{\sigma}^{i}=\right.$ boundary of $\left.\sigma^{i}\right)$,

(c) $g_{A}$ is fiber homotopic to $g$ fixed on the boundary, extend $g_{A}$ to all of $X$ by fiber homotopy extension.

We now prove a lemma which allows us to extend from $X_{A}^{i-1}$ to $X^{i}$. Note that this lemma also completes the proof of (2.4) since we can begin our induction by applying it in the case $i=0, X_{A}^{-1}=K_{A}^{-1}=\varnothing$.

(2.10) Lemma. Let $X_{A}^{i-1}$ be an annular neighborhood of $X^{i-1}$. Let $K_{A}^{i-1} \subset E$ be a compact set with $g\left(X_{A}^{i-1}\right) \subset K_{A}^{i-1}$. There exists $K^{i} \subset E$ with $K_{A}^{i-1} \subset K^{i}$ and $K^{i}$ compact, and a homotopy $g \sim \bar{g}$ over $f$ fixed on $X^{i-1}$ and such that $\bar{g}\left(X^{i}\right) \subset K^{i}$.

Proof. We begin by defining $K^{i}$. For each $k$, let $K_{k}^{i-1}=K_{A}^{i-1} \cap P^{-1}\left(U_{k}\right)$. Let $L_{k}^{i-1}=H_{k}\left(K_{k}^{i-1} \times I\right) \subset P^{-1}\left(U_{k}\right)$. Then $L_{k}^{i-1}$ is compact.

Now for each $k$, let $M_{k}^{i-1}=\phi_{k}\left(K_{k}^{i-1}\right) \subset U_{k} \times F$. Since $M_{k}^{i-1}$ is compact, there exists an integer $m_{k}$ with $M_{k}^{i-1} \subset U_{k} \times F^{m_{k}}$. Let $n_{k}=\max \left(i, m_{k}\right)$ and let $N_{k}^{i-1}=\psi_{k}\left(U_{k} \times F^{n_{k}}\right)$. Since $F$ is of finite type, $N_{k}^{i-1}$ is compact.

Let $K^{i}=K_{A}^{i-1} \cup\left(\cup_{k} L_{k}^{i-1}\right) \cup\left(\cup_{k} N_{k}^{i-1}\right)$.

We now deform $g$ over each $i$ cell $\sigma$ of $X$. Let $g(\sigma) \subseteq P^{-1}\left(U_{k}\right)$. Let $\bar{\sigma}_{1 / 2} \subset \bar{\sigma}$ be the interior $i$ cell of radius $<\frac{1}{2}$. Consider the map $\phi_{k} g: \sigma_{1 / 2} \rightarrow U_{k}$ 
$\times F$. By cellular approximation we may fiberwise deform this map into $\tilde{g}$ : $\bar{\sigma}_{1 / 2} \rightarrow U_{k} \times F^{n_{k}}$ leaving the map fixed on $\dot{\sigma}_{1 / 2}$.

Let $\bar{g}=\psi_{k} \tilde{g}$.

(a) $\bar{g}: \bar{\sigma}_{1 / 2} \rightarrow N_{k}^{i-1}$,

(b) $\bar{g}$ is fiberwise homotopic to $\psi_{k} \phi_{k} g$ on $\bar{\sigma}_{1 / 2}$ leaving $\dot{\sigma}_{1 / 2}$ fixed.

We now extend $\bar{g}$ to a map on all of $\sigma$ by setting

$$
\bar{g}(x)=H\left(g(x),|1-\|x\|| / 1-\frac{1}{2} \mid\right), \quad \frac{1}{2} \leqslant\|x\|<1 .
$$

One checks

(1) $\bar{g}=g / \dot{\sigma}$;

(2) if $\frac{1}{2} \leqslant\|x\| \leqslant 1, \bar{g}(x) \in L_{k}^{i-1}$, hence $\bar{g}(\sigma) \subseteq K^{i}$,

(3) $\bar{g}$ is fiberwise homotopic to $g$ leaving $\dot{\sigma}$ fixed.

3. The bounded lifting lemma. We will now show that the results of the previous section hold for $X$ finite dimensional normal. We will need the following lemmas.

(3.1) LEMMA. Let $U$ be a locally finite open cover of a normal space $X$, let $\nu U$ denote the nerve of $U$ and let $\pi_{U}: X \rightarrow \nu U$ be the "numeration" map [5,p. 355] of $X$ to the nerve. If $P$ is a simplicial complex and $\phi, \gamma: \nu U \rightarrow P$ are maps such that $\phi \pi_{U}$ and $\gamma \pi_{U}$ are homotopic, then there exists a locally finite open refinement $V$ of $U$ such that if $\pi_{U}^{V}: \nu V \rightarrow \nu U$ is a cannonical projection
(a) $\phi \pi_{U}^{V} \sim \gamma \pi_{U}^{V}$
(b) $\operatorname{dim} \nu V \leqslant \operatorname{dim} X$ (dim = covering dimension).

Proof. (a) $[15,4.2]$.

(b) In [7, Theorem 3.5] (or see [17, p. 132]) Dowker shows that every locally finite open cover of an $n$-dimensional normal space has an open refinement whose nerve has dimension $\leqslant n$. Since (a) is unaffected by refinement, we have (b).

(3.2) LeMma. For every map $f: X \rightarrow B$, with $X$ in $N$ :

(1) if $B$ is in $\tilde{W}$, there is a locally finite open cover $U$ of $X$ and a map $\phi$ : $\nu U \rightarrow B$ such that $f \sim \phi \pi_{U}$; and

(2) if $B$ is a finite simplicial complex, then $f \sim_{\beta} \phi \pi_{U}$.

Proof. Clearly (1) only depends on the homotopy type of $B$ so we may choose $B$ to be a simplicial complex. The result is now standard (see $[5, \mathrm{p}$. $356]$ or $[15,4.1])$.

(2) Since $\phi \pi_{U}$ and $f$ are in fact contiguous, the result follows from (1.9).

(3.3) Theorem (Bounded LifTing Lemma). A fibration $p: E \rightarrow B$ with $B$ in $\tilde{W}$ and fiber $F$ in $\tilde{W}_{f t}$ has the $\operatorname{BLP}\left(N_{f d}\right)$.

Proof. First, by (2.5) we can assume that $B$ is a finite complex. Suppose 
$X \in N_{f d}$ and the following diagram commutes:

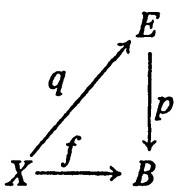

Since both $F$ and $B$ are in $\tilde{W}, E$ is also [18]. Hence, by (3.2) we can choose a locally finite open cover $U$ of $X$ and maps $\phi: \nu U \rightarrow B$ and $\gamma: \nu U \rightarrow E$ such that $\phi \rho_{U} \sim_{\beta} f$ and $\gamma \pi_{U} \sim g$. (Apply (3.2) to $E$ and $B$ and choose a common refinement.)

Since $p \gamma \pi_{U} \sim p g=f \sim \phi \pi_{U}$ we may apply (3.1) to obtain a locally finite open refinement $V, \operatorname{dim} \nu V \leqslant \operatorname{dim} X$ such that $p \gamma \pi_{U}^{V} \sim \phi \pi_{U}^{V}$. Hence, $\phi \pi_{U}^{V}$ : $\nu V \rightarrow B$ has a lift $\gamma^{\prime}: \nu V \rightarrow E$; thus by (2.4) there is a bounded map $\bar{\gamma}$ : $\nu V \rightarrow E$ with $\bar{\gamma}$ homotopic to $\gamma^{\prime}$ over $\phi \pi_{U}^{V}$.

Again, by (1.9) $\phi \pi_{U}^{V} \pi_{V} \sim_{\beta} \phi \pi_{U} \sim_{\beta} f$; hence, we can use the covering homotopy property with initial position $\beta\left(\bar{\gamma} \pi_{V}\right)$ to get a bounded lift $\bar{g}$ : $X \rightarrow E$ such that $p \bar{g}=f$.

We also have

$$
g \sim \gamma \pi_{U} \sim \gamma \pi_{U}^{V} \pi_{V} \sim \gamma^{\prime} \pi_{V} \sim \bar{\gamma} \pi_{V} \sim \bar{g} .
$$

Unfortunately we do not know $g \sim \bar{g}$ over $f$. To do this it would suffice to know the following:

Let $H_{1}$ be the homotopy $p \gamma \pi_{U} \sim p g=f$.

Let $H_{2}$ be the homotopy $p \gamma \pi_{U}^{V} \sim \phi \pi_{U}^{V}$.

Let $H_{3}$ be the homotopy $\phi \pi_{U} \sim f$.

Let $H_{4}$ be the homotopy $\pi_{U}^{V} \pi_{V} \sim \pi_{U}$. Now consider the following two compositions:

$$
\begin{gathered}
p \gamma \pi_{U}^{V} \pi_{V} \sim_{p \gamma H_{4}} p \gamma \pi_{U} \sim_{H_{1}} f \\
p \gamma \pi_{U}^{V} \pi_{V} \sim_{H_{2}\left(\pi_{V} \times \text { Id }\right)}^{\phi} \phi \pi_{U}^{V} \pi_{V} \sim_{\phi H_{4}} \phi \pi_{U} \sim_{H_{3}} f .
\end{gathered}
$$

If we knew that these two compositions were themselves homotopic with end maps fixed, we would be able to deform the homotopy $g \sim \bar{g}$ to one over $f$. This is the case, since careful examination of Morita's proof $[15,4.2]$ shows that $H_{2} \pi_{V}$ is contiguous to $H_{1}$.

4. The map $\beta^{*}$. In this section the Bounded Lifting Lemma is applied to the study of the function $\beta^{*}:[\beta X, Y] \rightarrow[X, Y]$.

(4.1) LemMa. Let $X$ be finite dimensional normal and $Y$ the homotopy type of a $C W$-complex of finite type; then $\beta^{*}$ is onto.

Proof. Clearly the result only depends on the homotopy type of $Y$, so we may assume $Y \in W_{f t}$. By (3.1) and (3.2), for each map $f: X \rightarrow Y$ there is a 
finite dimensional simplicial complex $Q$ and maps $\pi: X \rightarrow Q$ and $\phi: Q \rightarrow Y$ such that $\phi \pi \sim f$. Since $Q$ is finite dimensional and $Y$ is of finite type, we may deform $\phi$ into a cellular map $\bar{\phi}$ which is bounded. Thus $\beta^{*}[\beta(\bar{\phi} \pi)]=[\bar{\phi} \pi]=$ [f]. ([ ] denotes homotopy class.)

(4.2) THeOREM. Let $X$ and $Y$ be as in (4.1). Suppose $\pi_{1}(Y)$ is finite. Then $\beta^{*}$ : $[\beta X, Y] \rightarrow[X, Y]$ is a bijection.

PRoof. By (4.1) we need only show $\beta^{*}$ is 1-1. Again assuming $Y \in W_{f t}$, $\Omega Y \rightarrow Y^{I} \rightarrow^{p} Y \times Y$ is a fibration in the sense of (2.1). Since $\pi_{1}(Y)$ is finite and $Y$ is of finite type, $\Omega Y$ is also of finite type (see [21]) so we may apply the Bounded Lifting Lemma to this fibration.

Suppose $\bar{f}, \bar{g}: \beta X \rightarrow Y$ are such that $\beta^{*}[\bar{f}]=\beta^{*}[g]$. Let $f=\bar{f}|X, g=\bar{g}| X$. Since $\bar{f}$ and $\bar{g}$ are necessarily bounded, so are $f, g$ and $(f, g): X \rightarrow Y \times Y$.

Finally, since $f \sim g$ we have a lift $h: X \rightarrow Y^{I}$ with $p h=(f, g)$.

Applying the Bounded Lifting Lemma, we have a bounded lift $\bar{h}: X \rightarrow Y^{I}$ with $p \bar{h}=(f, g)$. Thus by $(1.5)$ we have $f \sim_{\beta} g$ or $[\bar{f}]=[\bar{g}]$.

Before going further, we give an application of (4.2). In particular, there is the following variant of the Borsuk Dowker homotopy extension theorem, in which we replace the condition that $X$ be binormal by the condition that $Y$ have a finite fundamental group. (For other versions without binormality, see [16].)

(4.3) Corollary. Let $A$ be a closed subspace of $X \in N_{f d}$. Suppose $Y$ is a compact $A N R$ with $\pi_{1}(Y)$ finite. Let $f, f^{\prime}: A \rightarrow Y$ be homotopic maps. Then if $f$ can be extended to a map $F: X \rightarrow Y$ then $f^{\prime}$ can be extended to $F^{\prime}: X \rightarrow Y$ with $F \sim F^{\prime}$.

Proof. From [14] and [21] we know $Y \in W_{f}$, so by (4.2), $\beta(f) \sim \beta\left(f^{\prime}\right)$. The result is now Borsuk's homotopy extension theorem applied to $(\beta X, \beta A)$ and $\beta(F)$.

We now consider the case $\pi_{1}(Y)$ not finite.

In general, (see (1.7)), $\operatorname{ker} \beta^{*}$ is not zero. In fact, the computation of (1.7) generalizes [3] to show that if $\pi_{1}(Y)$ contains an element of infinite order, then $\operatorname{ker} \beta^{*} \neq 0$. An interesting sidelight of this is that were the Burnside conjecture (every infinite finitely presented group has an element of infinite order) known to be true then (4.2) would be "best possible" for $Y \in W_{f t}$. If we assume $Y$ is also an $H$-space, then this question does not exist, and when $Y$ is a loop space, we are able to give a complete description of $\operatorname{ker} \beta^{*}$. We now consider that case.

(4.4) LeMma. Let $Y \in \tilde{W}_{f t}$ be an $H$-space. Let $d$ be the minimum number of generators of the free group $\pi^{1}(Y)=\left[Y, S^{1}\right]$. Let $T^{d}=S^{1} \times \cdots \times S^{1}$, d-times, be the d-torus. Then there exists a fibration $Y^{\prime} \rightarrow \tilde{Y} \rightarrow{ }^{h} T^{d}$ with 
section s: $T^{d} \rightarrow \tilde{Y}$, such that $\tilde{Y}$ has the homotopy type of $Y$. The fiber $Y^{\prime} \in \tilde{W}_{f t}$ with $\pi_{1}\left(Y^{\prime}\right)$ finite and $h$ is an $H$-space homomorphism (H-map).

Proof. Let $g_{1}, \ldots, g_{d}: Y \rightarrow S^{1}$ represent a set of generators of $\pi^{1}(Y)$. Define $\bar{h}=\left(g_{1}, \ldots, g_{d}\right): Y \rightarrow T^{d}$. Then $\bar{h}$ is an $H$-map. Since $Y$ is an $H$-space, $\pi^{1}(Y) \cong$ Free part of $\pi_{1}(Y)$. Let $\gamma_{1}, \ldots, \gamma_{d}: S^{1} \rightarrow Y$ represent the elements of $\pi_{1}(Y)$ corresponding to $g_{1}, \ldots, g_{d}$. Let $\bar{s}=\left(\gamma_{1} \times \cdots \times \gamma_{d}\right)$ : $T^{d} \rightarrow Y$.

Let $h: \tilde{Y} \rightarrow T^{d}$ be a fibration homotopy equivalent to $\bar{h}$ and let $e: Y \rightarrow \tilde{Y}$ be homotopy equivalence. Then $h$ is an $H$-map and has a section $s=e \bar{s}$. Let $Y^{\prime}$ be the fiber of $h$.

Because of $s$, the homotopy sequence for $h$ splits into short exact sequences giving $\pi_{n}\left(Y^{\prime}\right) \cong \pi_{n}(Y)$ for $n \neq 1$ and a short split exact sequence

$$
0 \longrightarrow \pi_{1}\left(Y^{\prime}\right) \longrightarrow \pi_{1}(Y) \stackrel{\bar{h}_{\#}}{\stackrel{\bar{s}_{\#}}{\rightleftarrows}} \pi_{1}\left(T^{d}\right) \longrightarrow 0
$$

From the construction of $h, \pi_{1}\left(Y^{\prime}\right)$ is isomorphic to the torsion subgroup of $\pi_{1}(Y)$ which is finite. Now $Y^{\prime} \in \tilde{W}$ since $Y$ and $T^{d} \in \tilde{W}$; hence $Y^{\prime} \in W_{f t}$ with $\pi_{1}\left(Y^{\prime}\right)$ finite.

(4.5) TheOREM. Let $Y$ be a loop space in $\tilde{W}_{f}$. Then we have the following commutative diagram of groups and homomorphisms with rows and columns exact:

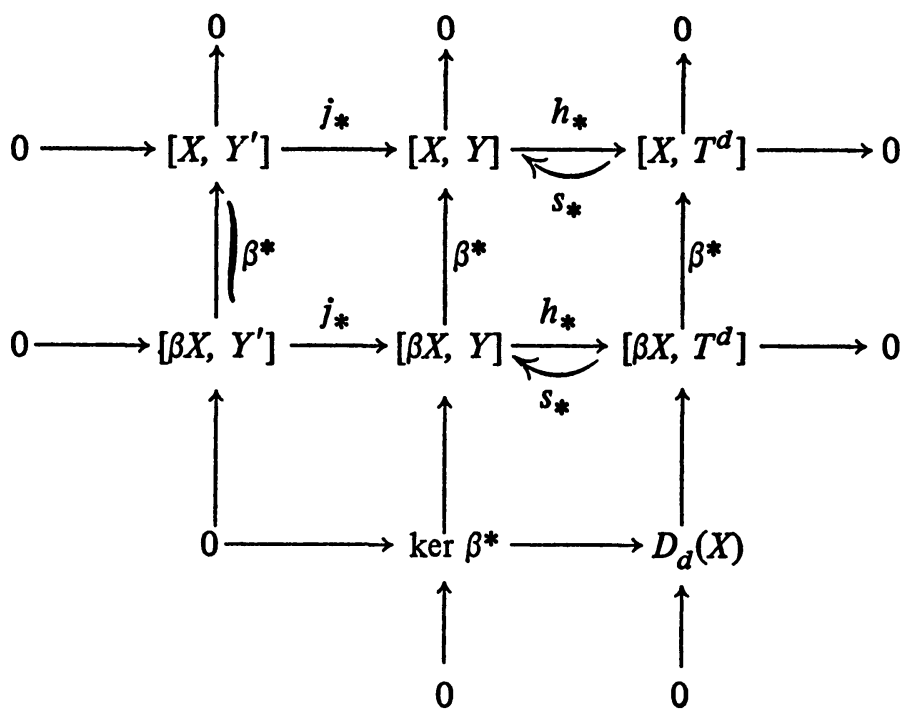

Proof. The group structures on $\left[X, Y^{\prime}\right]$ and $\left[\beta X, Y^{\prime}\right]$ are the ones induced by "inclusion" $j: Y^{\prime} \rightarrow Y . h_{*}$ is a homomorphism since $h$ is an $H$-map, $s_{*}$ is a 
homomorphism because it splits the sequence. $\beta^{*}:\left[\beta X, Y^{\prime}\right] \rightarrow\left[X, Y^{\prime}\right]$ is an isomorphism by (4.2).

From the diagram we see that

$$
\operatorname{ker}\left\{\beta^{*}:[\beta X, Y] \rightarrow[X, Y]\right\} \cong D_{d}(X)=\operatorname{ker}\left\{\beta^{*}\left[\beta X, T^{d}\right] \rightarrow\left[X, T^{d}\right]\right\}
$$

But from (1.7), $D_{d}(X)=\bigoplus_{d} C(X) / C^{*}(X)$, and since $C(X) / C^{*}(X)$ is a torsionless divisible abelian group, it is isomorphic to a direct sum of $Q$ 's (the rationals). So finally, we have the following:

(4.6) THEOREM. For $Y \in \tilde{W}_{f t}$ a loop space and $X \in N_{f d}$, we have the following split exact sequence:

$$
0 \rightarrow \underset{\alpha}{\oplus} Q \rightarrow[\beta X, Y] \stackrel{\beta^{*}}{\rightarrow}[X, Y] \rightarrow 0
$$

We denote $\oplus_{\alpha} Q=\operatorname{ker} \beta^{*}$ as $D(X, Y)$.

5. The cohomotopy of Stone-Čech remainders. The Stone-Čech remainder, $N X=\beta X-X$, of a space $X$ has been studied quite extensively (see for example [22]). General topological methods have not proved too successful in deciding questions of when $N X$ is not homeomorphic to $N Y$ from the properties of $X$ and $Y$. In [1] and [11] we, separately, had some success with a problem of this kind by considering the cohomotopy properties of $N X$, and were thus able to settle a question about locally compact groups raised by $\mathrm{J}$. P. Kahane.

In this section, we update the investigations initiated in [1], [2] and [11] by giving a complete description of $[N X, Y]$ in terms of $X$ and $Y$ under reasonable hypotheses on $X$ and $Y$.

If $X$ is locally compact then it is immediate that

$$
N X=\bigcap_{\alpha \in A}\left(\beta X-\text { int } K_{\alpha}\right)
$$

where $\left\{K_{\alpha}: \alpha \in A\right\}$ is the set of all compact subsets of $X$ and int $K_{\alpha}$ is the interior of $K_{\alpha}$ in $X$. Thus if $Y$ has the homotopy type of an ANR or equivalently the homotopy type of a countable $C W$-complex [14], a simple direct limit argument shows that

$$
[N X, Y]=\text { ind } \lim \left\{\left[\beta X-\operatorname{int} K_{\alpha}, Y\right]: \alpha \in A\right\},
$$

where the ordering is induced by inclusion maps. But $\beta X-$ int $K_{\alpha}=\beta(X-$ int $K_{\alpha}$ ) and so (4.2) yields the following

(5.1) THEOREM. Let $X$ be a locally compact space in $N_{f d}$ and let $Y \in \tilde{W}_{f t}$ with $\pi_{1}(Y)$ finite; then

$$
[N X, Y] \cong \text { ind } \lim \left\{\left[X-\operatorname{int} K_{\alpha}, Y\right]: \alpha \in A\right\} .
$$

Using (5.1) we are able to give the following relation between the 
cohomotopy of the boundary of a manifold and its Čech boundary.

(5.2) COROLlary. Let $M^{n}$ be a compact manifold with boundary $\partial M^{n}$ and let $Y \in \tilde{W}_{f t}$ with $\pi_{1}(Y)$ finite. Then

$$
\left[\partial M^{n}, Y\right] \cong\left[N\left(M^{n}-\partial M^{n}\right), Y\right] .
$$

Proof. $M^{n}-\partial M^{n}$ is locally compact and let $\partial M^{n} \times[0,1]$ be a tubular neighborhood of $\partial M^{n}$ in $M^{n}$; then $M^{n}-\left(\partial M^{n} \times[0,1 / k)\right)$ is a compact subset $K_{k}$ of $M^{n}$ for each positive integer $k$. Let $\dot{M}^{n}=M^{n}-\partial M^{n}$. Then $\left\{\dot{M}^{n}-\right.$ int $\left.K_{k}\right\}$ is cofinal in $\left\{\dot{M}^{n}-\right.$ int $\left.K_{\alpha}: \alpha \in A\right\}$, and the inclusion $\dot{M}^{n}-$ int $K_{k} \rightarrow \dot{M}^{n}-$ int $K_{k^{\prime}}, k \geqslant k^{\prime}$, induces a bijection

$$
\left[\dot{M}^{n}-\text { int } K_{k}, Y\right] \cong\left[\dot{M}^{n}-\text { int } K_{k}, Y\right]
$$

and so

$$
\text { ind } \lim \left\{\left[\dot{M}^{n}-\text { int } K_{\alpha}, Y\right]: \alpha \in A\right\}=\left[\dot{M}^{n}-\text { int } K_{\alpha} ; Y\right]
$$

for any $\alpha$. But $\dot{M}^{n}-$ int $K_{k}$ is homotopy equivalent to $\partial M^{n}$ for each $k$.

(5.3) EXAMPLE 1. As a direct application of (5.2) we have

$$
\left[N R^{n}, Y\right] \cong \pi_{n-1}(Y) \text {. }
$$

This is because $N R^{n}=N\left(D^{n}-\partial D^{n}\right)$ for $D^{n}$ the closed $n$-disc.

Example 2. Let $C$ denote the Cantor set $\{0,1\}^{\omega}$. Let $L^{n}=D^{n} \times C-\{0$, c) where $c$ is any part of $C$. $L^{n}$ is locally compact, and by an analogous argument to that of (5.2) we have that

$$
\left[N L^{n}, Y\right] \simeq\left[L^{n}, Y\right]
$$

for all $Y \in \tilde{W}_{f t}$ with $\pi_{1}(Y)$ finite.

In [1] and [11] it was shown that there exist $Y^{n}$ for each $n$ such that [ $N L^{n}$, $\left.Y^{n}\right] \neq\left[N R^{n}, Y^{n}\right]$ and hence that $N L^{n} \neq N R^{n}$.

6. Čech and Kan extensions. In this section we observe that Theorem 4.3 also has implications in the study of the Čech and Kan extensions of representable functors. More particularly, we ask when the Čech extension (finite covers) of a homotopy functor is a homotopy functor. Since by Dowker's result (1.7), $\check{H}_{f}^{1}\left(R^{1}, Z\right)=C(R) / C_{*}(R)$, we know that, in general, Cech extensions are not homotopy functors. Below we show that on $N_{f d}$ this is again somewhat of an exceptional case. We also show that $\check{H}_{f}^{2}(X, Z)$ is a homotopy functor on all of $N$.

By the Kan extension of a functor we will mean right Kan extension along the inclusion. Given a space $B$ we consider various ways of extending the functor $[-, B]$ from one category to a larger one.

For $\overline{P_{l}}=$ the category of locally finite simplicial complexes and homotopy classes of maps and $\bar{N}=$ the category of normal spaces and homotopy classes of maps, we make the following definition: 
$\bar{h}_{B}^{K}=$ the Kan extension of $[-, B]$ from $\bar{P}_{l}$ to $\bar{N}$.

$\check{h}_{B}=$ the Čech extension to $N$ with respect to numerable covers.

$\check{H}_{B}=$ the Cech extension to $N$ with respect to finite covers.

$h_{B}^{K}=\bar{h}_{B}^{K} \circ R$, where $R: N \rightarrow \bar{N}$ is the quotient functor.

There are various relations between the above which we list in the form of a theorem.

(6.1) THEOREM. On $N$ there are the following natural equivalences among the various functors mentioned above:

1. $h_{B}^{K}=\check{h}_{B}=[-, B]$,

2. $\check{H}_{B}=\check{h}_{B} \beta$.

Hence:

3. $\check{H}_{B}=[\beta-, B]$.

Proof. 1. [5].

2. This is essentially [8, p. 282].

The representation of $\check{H}_{B}$ provided by (6.1)(3) allows immediate application of the results of $\$ 4$. In particular, we have

(6.2) THEOREM. Let $B \in \tilde{W}_{f t}$ with $\pi_{1}(B)$ finite, then on $N_{f}$ we have $\check{H}_{B}=\check{h}_{B}$ $=h_{B}^{K}$. In particular, $H_{B}$ is a homotopy invariant on $N_{f d}$.

Proof. The proof is an immediate corollary of $(6.1)(2)$ and the fact that under these hypotheses $[X, B]=[\beta X, B]$ (by (4.2)).

Since $\check{H}^{n}(; G)$, Čech cohomology with respect to numerable covers, is representable on Top by $K(G, n)$ [5]; we have the following

(6.3) COROLlaRY [3]. On $N_{f d}$ and with $G$ a finitely generated abelian group, $\check{H}^{n}(-; G)=\check{H}_{f}^{n}(-; G)$ ( finite covers) for $n>1$, and for $n=1$ with $G$ finite. In particular, $\check{H}_{f}^{n}(-, G)$ is a homotopy invariant under these hypotheses.

We next ask when $\check{H}_{B}=[\beta-, B]$ is a homotopy functor on all of $N$. One would expect that this is not often the case. Indeed, in [4] we showed that if $B$ is a finite complex with nonzero homology then $\check{H}_{B}$ is not a homotopy functor. On the other hand, we have the following

(6.4) THEOREM. Let $B$ be the homotopy type of a $C W$-complex such that $\Omega B$ has the homotopy type of a finite complex; then $\check{H}_{B}=[\beta-, B]$ is a homotopy functor.

Proof. This follows at once from the fact that under the hypothesis $\beta^{*}$ : $[\beta X, B] \rightarrow[X, B]$ is one-one. This in turn is proved as in (4.2) using the special form of the Bounded Lifting Lemma (2.3).

This theorem has the following surprising corollary.

(6.5) COROLlARY. $\check{H}_{f}^{2}(-; Z)$ is a homotopy functor on all of $N$. 
Proof. $\Omega K(Z, 2)=K(Z, 1)$ has the homotopy type of $S^{1}$.

(6.6) Final Remarks. First it should be observed that the proof of (6.5) actually shows $\check{H}_{f}^{2}(X, Z)$ to be a subgroup of $\check{H}^{2}(X ; Z)$. Of course, on $N$ they are not, in general, equal.

Corollary (6.5) raises the somewhat surprising possibility that for $n>1, \check{H}_{f}^{n}$ is a homotopy functor on $N$, as it is on $N_{f d}$. The special nature of the proof for $n=2$ suggests this is not the case. However, one would like to know to what extent (6.5) is a special case.

7. Speed and path length: Applications to manifolds.

(7.1) If $Y$ is a metric space with metric $\rho$ and $\sigma: I \rightarrow Y$ is a map, the path length or variation $|\sigma|$ of $\sigma$ is defined to be

$$
\sup \left\{\sum_{i=1}^{n} \rho\left(\sigma\left(t_{i-1}\right), \sigma\left(t_{i}\right)\right)\right\}
$$

over all sequences $0=t_{0} \leqslant t_{1}<t_{2} \leqslant \cdots<t_{n}=1$. If the set is unbounded, then $|\sigma|=\infty$.

(7.2) A homotopy $H: X \times I \rightarrow Y$ is said to have bounded path length if $\left\{\left|H_{x}\right|: x \in X\right\}$ is bounded, where $H_{x}: I \rightarrow Y$ is the path $H_{x}: I \rightarrow Y$ is the path $H_{x}(t)=H(x, t)$.

Note that if $Y$ is compact, this definition does not depend on the metric.

(7.3) A homotopy $H: X \times I \rightarrow Y$ is said to have bounded speed if $\{\rho(H(x$, $\left.\left.t), H\left(x, t^{\prime}\right)\right) /\left(t-t^{\prime}\right): x \in X, t, t^{\prime} \in I\right\}$ is bounded. In particular, if $H_{x}$ is differentiable for all $x \in X$, then $H$ has bounded speed if $\left\{\left|H_{x}^{\prime}(t)\right|: x \in X\right.$, $t \in I\}$ is bounded.

Recalling (1.3)(d) it would appear that the concepts of uniform, bounded path length and bounded speed for homotopies into compact spaces are very similar. In fact, bounded speed implies the other two but there are no other relations between the three properties. On the other hand, we shall show that for reasonable $Y$ the existence of a uniform homotopy between a pair of maps implies the existence of a uniform homotopy with bounded path length and speed between them.

(7.4) Lemma. $A$ homotopy $H: X \times I \rightarrow Y$ with bounded speed has bounded path length, and, if $Y$ is compact, $H$ is uniform.

Proof. By assumption there is a number $M$ such that $\rho(H(x, t), H(x$, $\left.\left.t^{\prime}\right)\right) \leqslant M\left|t-t^{\prime}\right|$ for all $x \in X$ and $t, t^{\prime} \in I$, so clearly $\left|H_{x}\right| \leqslant M$ for all $x$. If $Y$ is compact then $H$ is uniform by (1.3)(d).

(7.5) ExAMPLE. A homotopy of bounded path length which has unbounded speed and is not uniform.

Define $H:[0,1) \times I \rightarrow I \times I$ by 


$$
H(x, t)= \begin{cases}(x, 1), & t \leqslant x, \\ (x,((1-t) /(1-x))), & x<t .\end{cases}
$$

Clearly as $x$ approaches 1 , the speed of $H_{x}$ increases without bound. Also, if $x \in(t, 1], H(x, t)-H(x, 1)=1$ so $H$ is not uniform by (1.3)(d).

(7.6) Uniform homotopies with infinite path length are easy to construct, for instance $H: * \times I \rightarrow I$ given by $H(*, t)=t \sin (1 / t)$, where $*$ is a one point space.

One can also have uniform homotopies of unbounded path length in which $\left|H_{x}\right|$ is finite for each $x$. For example:

Let $S(t)=t \sin (1 / t), t \in I$. Let $h:\left\{(a, b) \in I^{2}: b<a\right\} \times I \rightarrow I$ be a $C^{\infty}$ function with

$$
h(a, b, t)= \begin{cases}0, & a \geqslant t \\ 1, & b \leqslant t\end{cases}
$$

Define $H:[2, \infty) \times I \rightarrow \times I$ by

$$
H(x, t)=\left(S(t), h\left(x^{-1},(x-1)^{-1}, t\right), 1-t\right) .
$$

Now $H$ is a $C^{\infty}$ function except at $t=1$ and is a homotopy from the constant function to $(0,1)$ to the constant function to $(\sin 1,0)$. Also as $x \rightarrow \infty, H_{x}(t) \rightarrow S(t)$ for all $t \in I$, so $\left|H_{x}\right| \rightarrow|S|=\infty$.

It remains only to show that $H$ is uniform. Since

$$
\begin{aligned}
& \left|H(x, t)-H\left(x, t^{\prime}\right)\right| \\
& \quad \leqslant\left|S(t) h\left(\frac{1}{x}, \frac{1}{x-1}, t\right)-S\left(t^{\prime}\right) h\left(\frac{1}{x}, \frac{1}{x-1}, t^{\prime}\right)\right|+\left|t-t^{\prime}\right|,
\end{aligned}
$$

we need only show that given $\varepsilon>0$ there exists $\delta>0$ such that $\left|t-t^{\prime}\right|<\delta$ implies that

$$
D_{x}\left(t, t^{\prime}\right)=\left|S(t) h\left(\frac{1}{x}, \frac{1}{x-1}, t\right)-S\left(t^{\prime}\right) h\left(\frac{1}{x}, \frac{1}{x-1}, t^{\prime}\right)\right|<\varepsilon .
$$

Clearly $D_{x}\left(t, t^{\prime}\right) \leqslant|S(t)|+\left|S\left(t^{\prime}\right)\right| \leqslant t+t^{\prime}$, so for $t \in[0, \varepsilon / 2]$, and $\left|t-t^{\prime}\right|$ $<\varepsilon / 2, D_{x}\left(t, t^{\prime}\right)<\varepsilon$.

Now for $x>c \geqslant 2 / \varepsilon+1, h\left(-x^{-1},(x-1)^{-1}, t\right)=1$ for all $t \in[\varepsilon / 2,1]$. So for $x \geqslant c$ and $t, t^{\prime} \in[\varepsilon / 2,1]$,

$$
D_{x}\left(t, t^{\prime}\right)=\left|S(t)-S\left(t^{\prime}\right)\right|
$$

But $S$ is uniformly continuous so there exists $\delta^{\prime}>0$ such that $\left|t-t^{\prime}\right|<\delta^{\prime}$ implies $D_{x}\left(t, t^{\prime}\right)<\varepsilon$.

Lastly, since $[2, c] \times I$ is compact, there exists $\delta^{\prime \prime}>0$ such that $\left|t-t^{\prime}\right|<$ $\delta^{\prime \prime}$ implies $D_{x}\left(t, t^{\prime}\right)<\varepsilon$ for all $x \in[2, c]$.

Let $\delta=\min \left\{\varepsilon / 2, \delta^{\prime}, \delta^{\prime \prime}\right\}$. 
(7.8) Lemma. Let $Y$ be either a finite simplicial complex or a compact Riemannian manifold (with or without boundary). For any uniform homotopy $H$ : $X \times I \rightarrow Y$ there is a continuous family of uniform homotopies $H^{s}: X \times I \rightarrow$ $Y, s \in I$, such that:

(1) $H^{0}=H$.

(2) $H_{0}^{s}=H_{0}$ and $H_{1}^{s}=H_{1}$ for all $s \in I$.

(3) $H^{1}$ has bounded speed (and hence path length).

(4) If $X$ is a $C^{\infty}$ and $Y$ is a compact Riemannian manifold then $H^{1}$ is $C^{\infty}$ (except on $X \times\{0,1\}$ if $H$ is not $C^{\infty}$ on $X \times\{0,1\}$ ).

(5) If $X$ is a simplicial complex and $Y$ is a finite simplicial complex, then $H^{1}$ is piecewise linear $(P L)$ (except on $X \times\{0,1\}$ if $H$ is not $P L$ on $X \times\{0,1\})$.

Before proving (7.8) we observe that combining it with (4.2) gives

(7.9) THEOREM. Let $Y$ be a finite complex or a compact Riemannian manifold. If $\pi_{1}(Y)$ is finite and $X \in N_{f d}$, then any two maps $f, g: X \rightarrow Y$ which are homotopic are uniformly homotopic via a homotopy with bounded speed and path length.

Moreover if $X$ is a $C^{\infty}$-manifold (simplicial complex) the homotopy can be chosen to be $C^{\infty}$ (PL) except possibly at $f$ and $g$.

(7.10) Essential to our proof of (7.8) is the concept of an equilocally convex structure [11], [14].

Definition. We say $\left(U, \lambda,\left\{V_{\alpha}\right\}\right)$ is an equilocally convex structure on a space $Y$ if $U$ is a neighborhood of the diagonal of $Y \times Y, \lambda: U \times I \rightarrow Y$ satisfying $\lambda(a, b, 0)=a, \lambda(a, b, 1)=b$ and $\lambda(a, a, t)=a$, and $\left\{V_{\alpha}\right\}$ is an open covering of $Y$ such that $V_{\alpha} \times V_{\alpha} \subset U$ and $\lambda\left(V_{\alpha} \times V_{\alpha} \times I\right)=V_{\alpha}$ for all $\alpha$.

(7.11) LEMMA. Let $Y$ be either a finite complex or a compact Riemannian manifold. There exists a number $\varepsilon>0$ such that $Y$ admits an equilocally convex structure such that:

(a) $\left\{V_{\alpha}\right\}$ is finite and diameter $V_{\alpha} \leqslant \varepsilon$ for all $\alpha$.

(b) For $a, b \in V_{\alpha}, \lambda(a, b, t)$ is a geodesic path of length $\rho(a, b)$, $\rho$ being $a$ (Riemannian) metric on $Y$.

(c) If $Y$ is a $C^{\infty}$ manifold, then $\lambda$ is $C^{\infty}$.

The proof is an exercise in local Riemannian geometry or local simplicial geometry as the case may be.

(7.12) Proof of (7.8). Let $H: X \times I \rightarrow Y$ be a uniform homotopy. Let ( $U$, $\left.\lambda\left\{V_{\alpha}\right\}\right)$ be an equilocally convex structure for $Y$ satisfying (7.11). Let $\gamma$ be the Lebesgue number for the cover $\left\{V_{\alpha}\right\}$ and let $\delta>0$ be such that $\left|t-t^{\prime}\right|<\delta$ implies that $\rho\left(H(x, t), H\left(x, t^{\prime}\right)\right)<\delta$ for all $x \in X$. Finally, let $0=t_{0} \leqslant t_{1}$ 
$<\cdots<t_{n}=1$ be a finite sequence such that $\left|t_{i-1}-t_{i}\right|<\delta$ for all $i=$ $1, \ldots, n$.

We define $H^{1}$ piecewise. In particular, $H_{x}^{1} \mid\left[t_{i-1}, t_{i}\right]$ is the geodesic from $H\left(x, t_{i-1}\right)$ to $H\left(x, t_{i}\right)$. In formula,

$$
H^{1}(x, t)=\lambda\left(H\left(x, t_{i-1}\right), H\left(x, t_{i}\right), \frac{t-t_{i-1}}{t_{i}-t_{i-1}}\right)
$$

for $t \in\left[t_{i-1}, t_{i}\right]$ and all $x \in X$.

One verifies that $H^{1}$ is well defined and of bounded speed.

Define $H^{s}: X \times I \rightarrow Y$ by

$$
H^{s}(x, t)=\lambda\left(H(x, t), H^{1}(x, t), s\right) .
$$

That $H^{1}$ can be made $C^{\infty}(\mathrm{PL})$ follows from standard smoothing (PL-ing) techniques.

8. Obstruction theory for Stone-Čech compactifications. The question as to whether a given homotopy can be replaced by a uniform homotopy is a special case of the problem of completing the following diagrams.

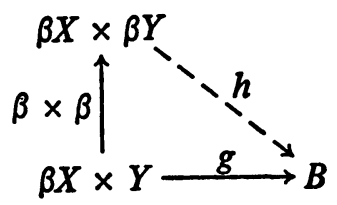

Even for $B=I$, one cannot, in general, expect to complete such a diagram so that it strictly commutes unless both $X$ and $Y$ are pseudocompact [12] which is not a condition that is of ten present.

In this section we consider an intermediate problem. Namely, for $X \in N_{f d}$, $B \in \tilde{W}_{f t}$ and $(Y, A)$, a relative $C W$-complex, when can one find $h$ to complete the following diagram?

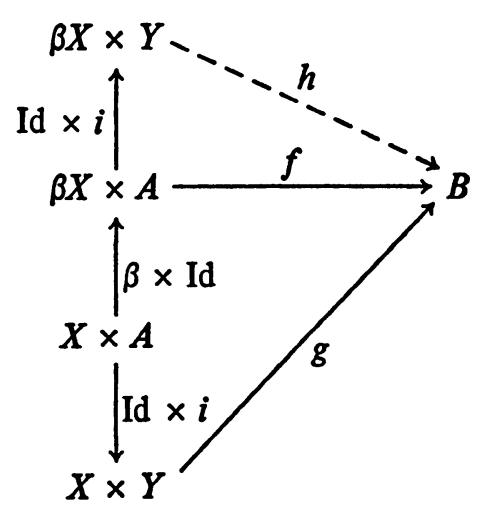


to be strictly commutative and such that the diagram

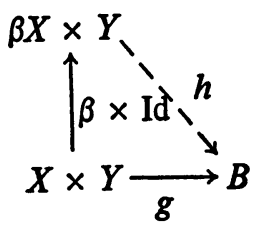

commutes up to homotopy $\bmod (X \times A)$.

Note that (4.3) says that if $\pi_{1}(B)$ is finite and $(Y, A)=(I,\{0,1\})$, then we can always find such an $h$. This allows the following straightforward generalization.

(8.3) THEOREM. Suppose $\pi_{i}(B)$ is finite for $i \leqslant \operatorname{dim}(Y, A)$; then $a$ map $h$ exists so that (8.1) and (8.2) commute as required.

Proof. (By induction on the skeleta of $(Y, A)$ ). Let $Y^{n}=A \cup$ (cells of $Y$ of $\operatorname{dim} \leqslant n)$. Assume a map $h_{i}: \beta X \times Y^{i} \rightarrow B$ satisfying the conditions (8.1)-(8.2) for the pair $\left(Y^{i}, A\right)$. We now wish to find $h_{i+1}$. We may do this cell by cell.

Let $\sigma$ be an $i+1$ cell of $Y-A$. Suppose we are given a map $f: \beta X \times \dot{\sigma} \rightarrow$ $B$ and an extension $g: X \times \sigma \rightarrow B$ of $f \mid X \times \dot{\sigma}$. We wish to know when we may extend $f$ to $h: \beta X \times \sigma \rightarrow W$ such that (8:1)-(8.2) holds. This is equivalent to the following problem. When can we complete the following diagram

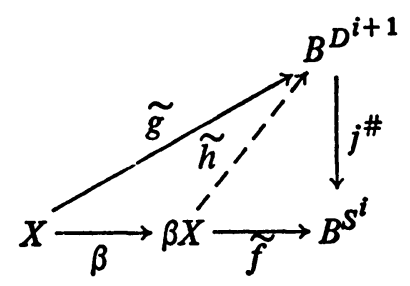

up to homotopy over $\tilde{f} \beta$ ?

Now $j^{\#}$ is a fibration with fiber $B^{S^{i+1}}$, and since $\pi_{k}(B)$ is finite, $k \leqslant n$ and we know $B^{S^{\prime+1}}$ is of finite type. Hence, $h$ exists by the Bounded Lifting Lemma (3.3).

We now consider the problem from another point of view. Namely, assuming $B$ is not as "nice" as (8.3). What conditions must be put on $(Y, A)$ to assure the existence of $h$ ? We give a classical obstruction theoretic answer to this question under the hypothesis that $X$ is locally compact (we need $B^{X \times Y}=\left(B^{X}\right)^{Y}$ ) and $B$ is a loop space (we need (4.5)).

We now address the problem from the point of view of obstruction theory. In order to do this, we again give a slightly different formulation of the basic problem. In particular, given 


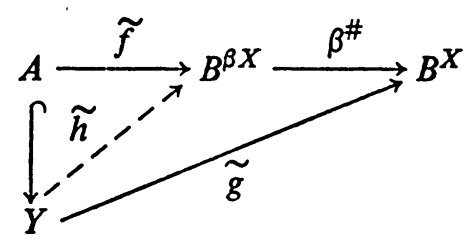

can we find $\tilde{h}$ with the appropriate commutivity relations?

The classical Eilenberg Obstruction [19] gives a formal answer to this question.

(8.5) Definition. Assume $B$ is a loop space; given (8.4) assume that we have extended $f$ to $Y^{i}$ such that

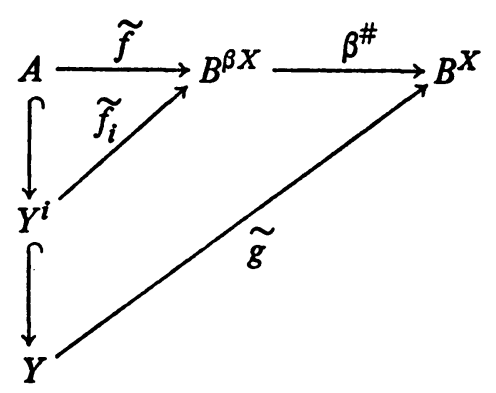

commutes on $A$ and homotopy commutes on $Y^{i}$.

We let $\tilde{\theta}^{i+1} \in C^{i+1}\left(Y, A ; \pi_{i}\left(B^{\beta X}\right)\right)$ be the $(i+1)$ th obstruction cocycle to extending $f$ to $Y^{i+1}$. Let $\beta_{i}^{*}: \pi_{i}\left(B^{\beta X}\right) \rightarrow \pi_{i}\left(B^{X}\right)$ be the map induced by $\beta$. Since $\beta_{i}^{*} \tilde{\theta}^{i+1}=0$ (by the existence of $\left.\tilde{g}\right)$ we may assume $\tilde{\theta}^{i+1}=C^{i+1}(Y, A$; ker $\left.\beta_{i}^{*}\right)$. Define $\theta^{i+1} \in H^{i+1}\left(Y, A ; \operatorname{ker} \beta_{i}^{*}\right)$ to be the corresponding cohomology class. We call $\theta^{i+1}$ the $(i+1)$ th obstruction to extending $\tilde{f}$ uniformly.

(8.6) THEOREM. ( $\left(\operatorname{ker} \beta_{i}^{*}\right)=\oplus_{\alpha} Q$, a direct sum of copies of $Q$. Hence, if $H^{i+1}(Y, A ; Z)$ is finite, $\theta^{i+1}=0$. In this case, $\tilde{f}_{i}$ may be extended to $Y^{i+1}$ (possibly modified on the $i$ cells of $Y^{i}-A$ ) so that $\beta^{\#} \tilde{f}^{i+1} \sim \tilde{g} \mid X \times Y^{i+1}$.

Proof. That $\left(\operatorname{ker} \beta_{i}^{*}\right)=\bigoplus_{\alpha} Q$ follows at once from (4.5) and the following commutative diagram:

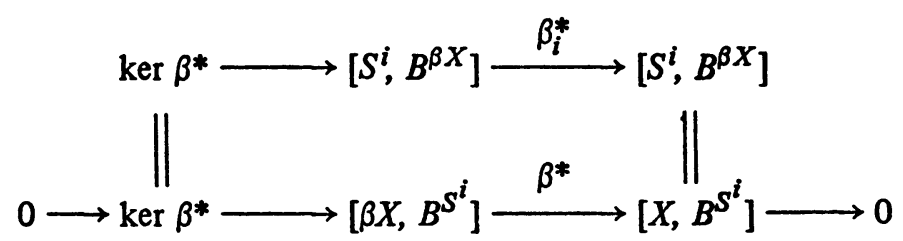

One should note that we are making implicit use of the fact that the various group structures are generated by the loop space structure of $B$. 
Thus $H^{i+1}(Y, A ; Z)$ finite implies $H^{i+1}\left(Y, A ; \operatorname{ker} \beta_{i}^{*}\right)=0$. Thus, $\theta^{i+1}=$ 0 . Thus, after possible modification on the $i$ cells by elements of $\operatorname{ker} \beta_{X}^{*}$ we can extend $\tilde{f}_{i}$ to $\bar{f}_{i+1}$.

We now construct $\tilde{f}_{i+1}$ from $\bar{f}_{i+1}$. Firstly, since we have modified $f_{i}$ by elements of $\operatorname{ker} \beta_{i}^{*}$ one can quickly check $\beta^{\#} \bar{f}_{i+1}\left|Y^{i} \sim g\right| Y^{i}$. It is possible that this relation does not hold with $Y^{i+1}$ replacing $Y^{i}$. In the language of obstruction theory, that the difference cochain $0 \neq\left[\beta^{\#} \bar{f}_{i+1}-\tilde{g}\right] \in C^{i+1}\left(Y^{i}\right.$; $\left.\pi_{i+1}\left(B^{X}\right)\right)$. But we can now use the fact that $\beta^{\#}$ is onto to modify $\bar{f}_{i+1}$ on the $i+1$ cells of $Y-A$ by this difference to a map $\tilde{f}_{i+1}$ so that

that is, $\beta^{\# \tilde{f}^{i+1}} \sim \tilde{\mathrm{g}} \mid X \times Y^{i+1}$.

$$
\left[\beta^{\#} \tilde{f}_{i+1}-\tilde{g}\right]=0 \in C^{i+1}\left(Y^{i} \cup A, \pi_{i+1}\left(B_{X}\right)\right),
$$

(8.6) has the following corollary.

(8.7) COROllaRY. In the above setting, suppose we are given a map $f$ : $\beta X \times A \rightarrow B$ and an extension of $f \mid X \times A$ to $g: X \times Y \rightarrow B$. Suppose $H^{k}(Y$, $A, Z)$ is finite for all $k$. Then there exists an extension of $f, h: \beta X \times Y \rightarrow B$, such that $h(\beta \times \mathrm{Id}) \sim g$.

\section{REFERENCES}

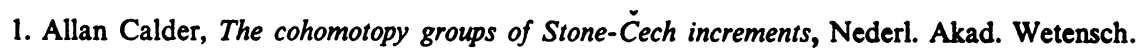
Proc. Ser. A 75 = Indag. Math. 34 (1972), 37-44. MR 46 \#2673.

2. __ On the cohomology of $\beta \mathbf{R}^{n}$, Quart J. Math. Oxford Ser (2) 25 (1974), 385-394. MR 51 \#1801.

3. __ Uniform homotopy, Fund. Math. (to appear).

4. A. Calder and J. Siegel, Homotopy and Kan extensions, Categorical Topology, Lecture Notes in Math., vol. 540, Springer-Verlag, Berlin and New York, 1976.

5. A. Dold, Lectures on algebraic topology, Springer-Verlag, Berlin and New York, 1972.

6. __ Partitions of unity in the theory of fibrations, Ann. of Math. (2) 78 (1963), 223-255. MR 27 \#5264.

7. C. H. Dowker, Mapping theorems for noncompact spaces, Amer. J. Math. 69 (1947), $200-242$. MR 8, 594.

8. S. Eilenberg and N. Steenrod, Foundations of algebraic topology, Princeton Univ. Press, Princeton, N. J., 1952. MR 14, 398.

9. L. Gillman and M. Jerison, Rings of continuous functions, Van Nostrand, Princeton, N. J., 1960. MR 22 \#6994.

10. I. Glicksberg, Stone- $\check{C}$ ech compactification of products, Trans. Amer. Math. Soc. 90 (1959), 369-382. MR 21 \#4405.

11. M. Jerson, J. Siegel and S. Weingram, Distinctive properties of Stone-C̈ech compactifications, Topology 8 (1967), 195-201. MR 39 \#607.

12. A. T. Lundell and S. Weingram, The topology of $\mathrm{CW}$ complexes, Van Nostrand, Princeton, N. J., 1969.

13. S. Mac Lane, Categories for the working mathematician, Springer-Verlag, Berlin and New York, 1971. MR 50 \# 7275.

14. J. Milnor, On spaces having the homotopy type of a CW-complex, Trans. Amer. Math. Soc. 90 (1959), 272-280. MR 20 \#6700.

15. K. Morita, Cech cohomology and covering dimension for topological spaces, Fund. Math. 87 (1975), 31-52. 
16. __ On generalizations of Borsuk's homotopy extension theorem, Fund. Math. 87 (1975), $1-6$.

17. A. R. Pears, Dimension theory of general spaces, Cambridge Univ. Press, London and New York, 1975.

18. J. Stasheff, A classification theorem for fibre spaces, Topology 2 (1963), 239-246. MR 27 \#4235.

19. E. H. Spanier, Algebraic topology, McGraw-Hill, New York, 1966. MR 35 \# 1007.

20. P. Tully, On regularity in Hurewitz fiber spaces, Trans. Amer. Math. Soc. 116 (1965), 126-134. MR 32 \#6463.

21. C. T. C. Wall, Finiteness conditions for CW-complexes, Ann. of Math. (2) 81 (1965), 56-69. MR 30 \# 1515.

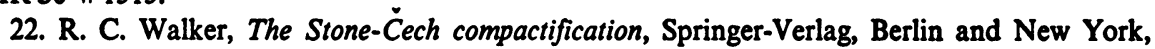
1974. MR 52 \#1595.

Department of Mathematics, Birkbeck College, london, England

Department of Mathematics, University of Missouri, St. Louis, Missouri 63121 